\title{
Eidgenössische Volksinitiative «Schutz vor Passivrauchen»
}

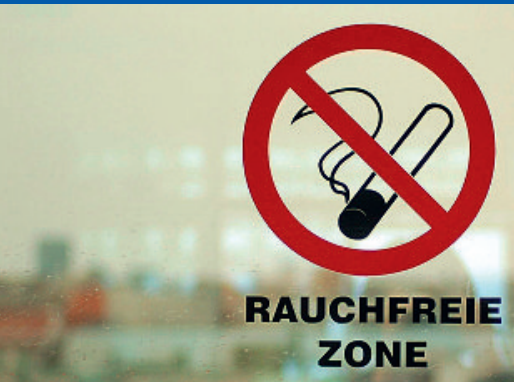

\section{Peter Wiedersheim}

Präsident der Ärztegesellschaft des Kantons St. Gallen

Helfen Sie mit,
wir zählen auf Sie!
Unterschriftsbögen
können bestellt
werden unter info@
rauchfrei-ja.ch oder per
Telefon: 0313782049.
Weitere Informationen
unter www.rauchfrei-ja.ch

Korrespondenz:

Dr. med. Peter Wiedersheim Ärztegesellschaft des Kantons St. Gallen

Rorschacherstrasse 150

CH-9006 St. Gallen

Tel. 0712450202

Fax 0712432771

ris@hin.ch
Die Delegiertenversammlung der FMH stellt sich mit grossem Mehr hinter die eidgenössische Volksinitiative «Schutz vor Passivrauchen»! Diese verlangt:

- Rauchfrei sind öffentlich zugängliche Räume, wie Restaurants, Bars, Schulen und Spitäler.

- Rauchfrei sind alle Arbeitsplätze in Innenräumen.

- In der ganzen Schweiz gilt die gleiche Regelung.

Am 27. September 2009 hat das Stimmvolk im Kanton St. Gallen mit grossem Mehr die Initiative «Schutz vor dem Passivrauchen für alle» angenommen. Die Initianten (Lungenliga St. Gallen, Krebsliga St. Gallen-Appenzell und die Ärztegesellschaft des Kantons St. Gallen) haben dies nicht zuletzt dank ihrer guten Zusammenarbeit erreicht. Umso unverständlicher war da das Abseitsstehen der FMH bei der inhaltlich identischen eidgenössischen Volksinitiative «Schutz vor Passivrauchen».

Nachdem sich die Konferenz der Ostschweizer Ärztegesellschaften (K-OCH), der Verband deutschschweizerischer Ärztegesellschaften (VEDAG) und die Konferenz der Kantonalen Ärztegesellschaften (KKA) einstimmig für die Unterstützung der Initiative der Allianz «Schutz vor Passivrauchen» entschieden hat, hat auch die Delegiertenversammlung der Schweizer Ärzteschaft FMH mit klarem Ja für die aktive Unterstützung der eidgenössischen Initiative «Schutz vor Passivrauchen» gestimmt. Sie hat sich damit im Sinn der Mehrheit der Schweizer Ärzteschaft entschieden.

\section{Warum ist diese Initiative nötig?}

Das ab 1. Mai 2010 geltende Bundesgesetz bringt keine einheitliche, umfassende Regelung für die ganze Schweiz. Die Kantone haben die Möglichkeit, konsequentere Regelungen zu erlassen. Bereits 15 Kantone kennen einen konsequenten Schutz vor Passivrauchen ohne Raucherlokale. Das Bundesgesetz ist deshalb heute schon überholt.

Es sieht auch keinen umfassenden Schutz der Arbeitnehmenden vor dem Passivrauchen vor:

- Restaurants und Bars mit einer Fläche von weniger als $80 \mathrm{~m}^{2}$ können als Raucherlokale geführt werden - das Service-Personal ist dem Tabakrauch permanent ausgesetzt.

- Gäste werden in Fumoirs vom Service-Personal bedient. Zudem sind Ausschankvorrichtungen in einem Fumoir erlaubt. Auch hier wird das ServicePersonal dementsprechend ständig dem Tabak- rauch ausgesetzt. Ein Restaurant kann die bestehende abgetrennte Bar zum Fumoir erklären.

- Das Bundesgesetz macht kaum Vorgaben zur Lüftung und Einrichtung von Raucherräumen. Es delegiert den Vollzug an die Kantone mit der Konsequenz der unterschiedlichen Regelungen.

- 15 Kantone kennen unterschiedlich weitergehende Regelungen als das Bundesgesetz. Die inhomogene Umsetzung führt zu wettbewerbsverzerrenden Verhältnissen in der Gastronomie, wie aber auch zu einer Verwirrung gerade für Touristinnen und Touristen.

- Die Volksinitiative «Schutz vor Passivrauchen» fordert genau das Gleiche, was in den Kantonen GE, VD, VS, NE, FR, BL, BS und SG vom Stimmvolk oder vom Parlament beschlossen wurde - sie entspricht also dem Volkswillen in vielen Kantonen. - Die konsequenten kantonalen Regelungen geraten durch das lückenhafte Bundesgesetz zunehmend unter Druck. In mehreren Kantonen, die bereits einen umfassenden Schutz vor Passivrauchen beschlossen haben, sind Bestrebungen im Gange, diesen rückgängig zu machen.

- Die Erfahrung im Ausland hat gezeigt, dass sich ein wirksamer Passivrauchschutz überall dort umsetzen liess, wo eine klare Regelung ohne Ausnahmen verfügt wurde (so z. B. in Irland, England, Norwegen, Finnland, Dänemark, Belgien, Frankreich, Italien, Schweden, Türkei usw.).

- Jährlich kostet Passivrauchen in der Schweiz über 400 Millionen Franken, verursacht 70000 Spitaltage und fordert 3000 verlorene Lebensjahre. $\mathrm{Zu}$ diesem Schluss kommt eine Studie unter der Leitung des Instituts für Sozial- und Präventivmedizin am Schweizerischen Tropeninstitut Basel, die erst kürzlich publiziert wurde.

Die obigen Fakten verlangen zwangsläufig ein geeintes und entschlossenes Auftreten der Ärzteschaft. Es gibt keine präventive Massnahme, die so viel bringt und so wenig kostet wie der wirksame Schutz vor dem Passivrauchen!

Der Zentralvorstand der FMH, aber auch alle Ärztinnen und Ärzte sind nun gefordert, mit einem aktiven Engagement die Volksinitiative «Schutz vor Passivrauchen» zu unterstützen und der bislang äusserst erfolgreichen Unterschriftensammlung zu einem fulminanten Endspurt zu verhelfen. 